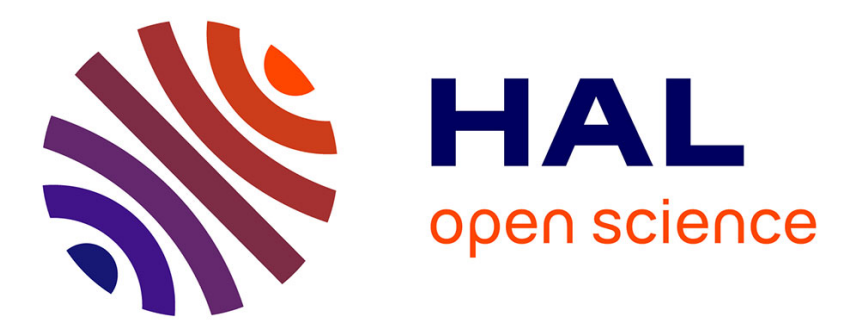

\title{
Towards a unified description of evaporation-residue fusion cross-sections above the barrier
} Philippe Eudes, Z. Basrak, F. Sébille, V. de La Mota, Guy Royer

\section{To cite this version:}

Philippe Eudes, Z. Basrak, F. Sébille, V. de La Mota, Guy Royer. Towards a unified description of evaporation-residue fusion cross-sections above the barrier. EPL - Europhysics Letters, 2013, 104, pp.22001. 10.1209/0295-5075/104/22001 . in2p3-00927530

\section{HAL Id: in2p3-00927530 https://hal.in2p3.fr/in2p3-00927530}

Submitted on 13 Jan 2014

HAL is a multi-disciplinary open access archive for the deposit and dissemination of scientific research documents, whether they are published or not. The documents may come from teaching and research institutions in France or abroad, or from public or private research centers.
L'archive ouverte pluridisciplinaire HAL, est destinée au dépôt et à la diffusion de documents scientifiques de niveau recherche, publiés ou non, émanant des établissements d'enseignement et de recherche français ou étrangers, des laboratoires publics ou privés. 


\title{
Towards a unified description of fusion evaporation-residue cross sections above the barrier
}

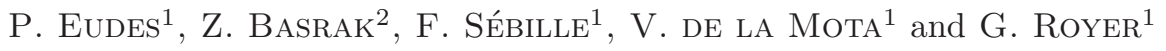 \\ 1 Subatech, EMN-IN2P3/CNRS-Université de Nantes, F-44307 Nantes, France \\ 2 Ruđer Bošković Institute, HR-10002 Zagreb, Croatia
}

PACS 25.70.Jj - Fusion and fusion-fission reactions

PACS 25.70.-z - Low and intermediate energy heavy-ion reactions

\begin{abstract}
A meticulous study of nearly 300 fusion-evaporation cross section data reveals that, when properly scaled, fusion excitation function complies with a universal homographic law which is, within experimental errors, reaction system independent. From a such complete and summed complete and incomplete fusion excitation functions extracted are the limiting energy for the complete fusion and the main characteristics (onset, maximum and vanishing) of the incomplete fusion. The DYWAN microscopic transport model correctly predicts the incomplete fusion cross section for incident energies $\gtrsim 15 \mathrm{~A} \mathrm{MeV}$ and suggests that the nuclear transparency is at the origin of fusion disappearance.
\end{abstract}

Introduction. - Since mid-seventies of the last century it is consensual that heavy-ion fusion reaction as a function of the center-of-mass energy passes through three distinct regions, that of rising (I), stagnating (II), and falling (III) cross sections, respectively. At low energies, the regions I and II have been systematically studied by confronting models with a myriad of experimental data [1-10]. In the region III, the incomplete fusion (IF) reaction mechanism opens and increasingly competes with the complete fusion (CF). To this region of fusion only two systematic analyses have been devoted by studying reactions leading to a rather light compound nucleus for which the formation of an evaporation residue is by far the main decay mode [11,12]. In the first study, Morgenstern et al. [11] have extracted the ratio of CF to CF + IF fusion cross sections showing that the energy of vanishing $\mathrm{CF}$ and that of the IF threshold strongly depend on the mass asymmetry of the system. In the second systematics, Lautesse et al. [12] have presented a fusion-evaporation excitation function relative to 7 light and quite symmetric systems including their own measurements around the Fermi energy of the $\mathrm{Ar}+\mathrm{Ni}$ and $\mathrm{Ni}+\mathrm{Ni}$ reactions. ${ }^{1}$ Thereby, $\mathrm{IF}$ process vanishes at incident energy of about $50 \mathrm{~A} \mathrm{MeV}$.

In line with previous studies, the goal of this letter is fourfold:

\footnotetext{
${ }^{1}$ The reported single-source cross sections of these two reactions are in the ensemble of collected fusion data the only ones which include both evaporation and multi-fragment channel contributions.
}

(i) Establish the first exhaustive systematics of $\mathrm{CF}$ as well as of CF + IF fusion-evaporation cross sections in region III.

(ii) Assess the global characteristics of fusion excitation function and reveal to which extent one may render it independent of reaction system.

(iii) Extract the main characteristics of both $\mathrm{CF}$ and IF components of fusion.

(iv) Testify that the fusion excitation function can be an interesting observable to constrain microscopic transport codes.

Being focused on the region III of fusion we restrict our search of fusion data at incident energy higher than about $3 A-4 A \mathrm{MeV}$. From the published reports we collected 238 total fusion-evaporation cross sections belonging to 48 reaction systems and additional $60 \mathrm{CF}$ data points belonging to 14 of the above systems with explicitely extracted both $\mathrm{CF}$ and IF cross sections. Data span over system mass $A_{\mathrm{sys}}=A_{\mathrm{trg}}+A_{\mathrm{prj}}=26-164$ nucleons $\left(A_{\mathrm{trg}}\left(A_{\mathrm{prj}}\right)\right.$ denotes target (projectile) mass number), mass asymmetry $\alpha=\left|A_{\text {trg }}-A_{\text {prj }}\right| /\left(A_{\text {trg }}+A_{\text {prj }}\right)=0-0.512$, and neutronto-proton content $N_{\text {sys }} / Z_{\text {sys }}=1-1.429$. Despite a broad coverage in the $\alpha$ and $N_{\mathrm{sys}} / Z_{\mathrm{sys}}$ variables many of data are for the mass symmetric systems and, in particular, for the systems with the equal number of neutrons $N_{\text {sys }}$ and protons $Z_{\text {sys }}$. About 100 additional data points from those measurements which have reported existence of a non-negligible fusion-fission component will be presented 


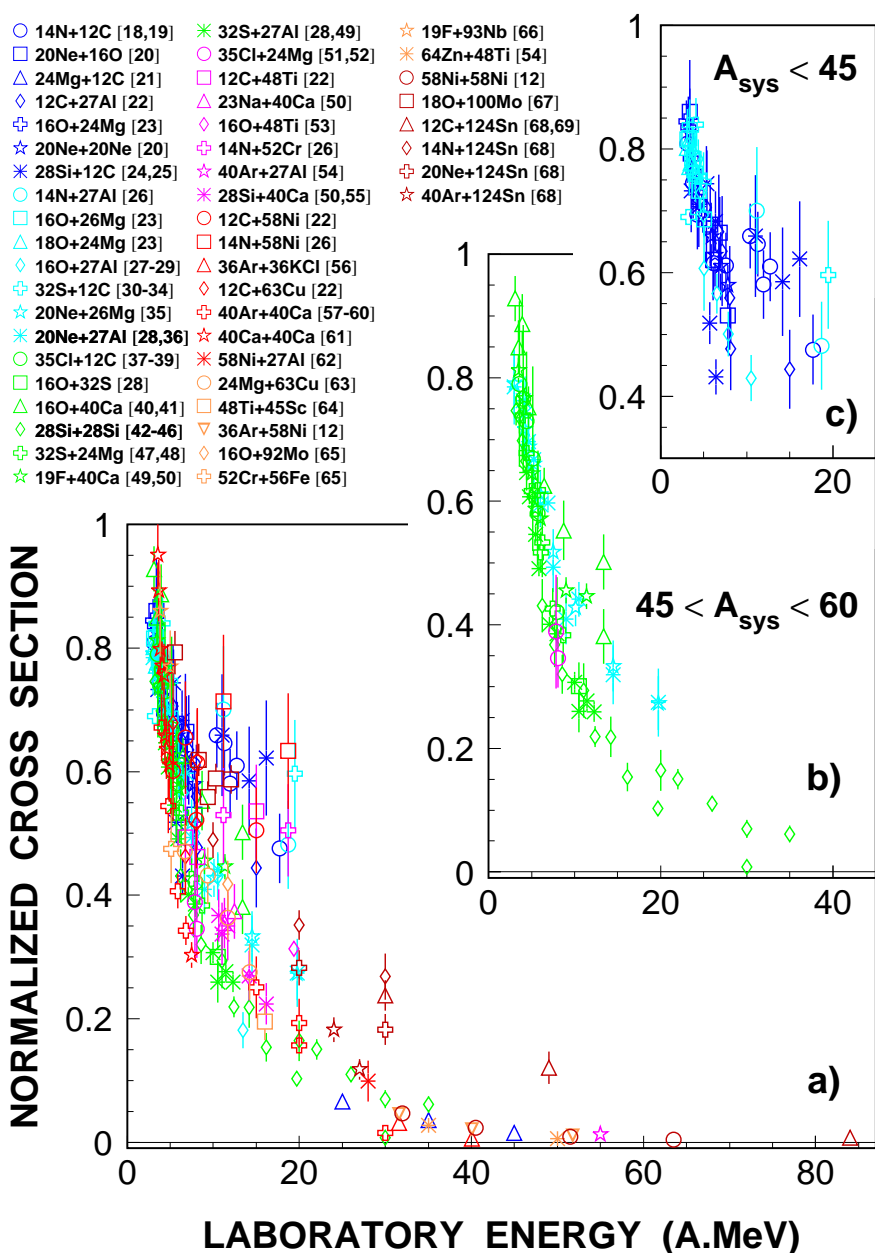

Fig. 1: (Color online.) Normalized fusion cross sections $\sigma_{N}$ plotted as a function of laboratory energy per nucleon $E_{\text {inc }}$. In panel a) are plotted all CF + IF data points from those works which have claimed that the fusion-fission component either does not exist or is negligible. To better resolve the stacked points in panels c) and b) are repeated subsets of the data for $A_{\text {sys }} \leq 44$ and $46 \leq A_{\text {sys }} \leq 59$, respectively. The inventoried systems are distinguished among them by symbols and a color code.

in a subsequent and more extensive work [13] but with thus enlarged data set our results and conclusions rest unaltered. The preliminary results of this work have been presented earlier [14].

Fusion excitation function. - In order to compare the fusion cross sections $\sigma_{F}$ belonging to so different reaction systems it is mandatory to account for the proportionality of $\sigma_{F}$ to the size of the system by renormalizing $\sigma_{F}$ by the (total) reaction cross section $\sigma_{R}$ at the same incident energy $E_{\text {inc }}=E_{\text {lab }} / A_{\text {prj }}$. We extensively studied the reliability of results for four parameterizations of $\sigma_{R}[2,15-17]$ and their influence on our investigation [13]. The two most recent of these empirical formulas give the most and about equally convincing predictions for $\sigma_{R}$ over the full domain of fusion data with respect to $E_{\text {inc }}, A_{\text {sys }}, \alpha$, and $N_{\text {sys }} / Z_{\text {sys }}$.

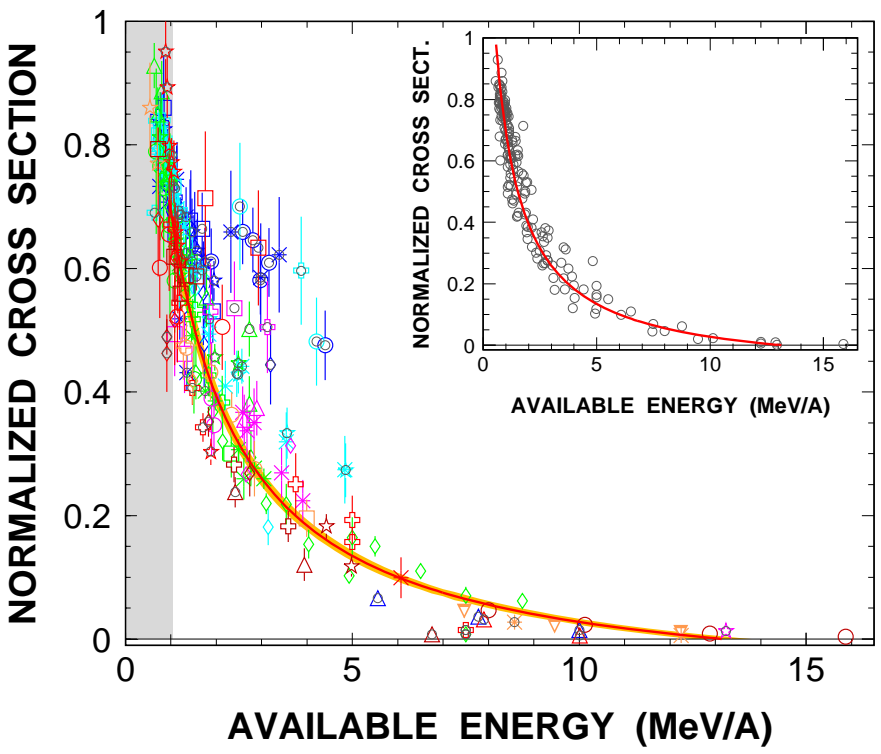

Fig. 2: (Color online.) $\sigma_{N}$ as a function of $E_{\text {avail }}$. The meaning of symbols and colors may be inferred from fig. 1 . The fit with the homographic function (eq. (4)) is shown as full red curve in both main panel and inset. The background band around the best fit curve is due to the errors on the fit parameters provided by the code used [70], see also in table 1 . Small circles are drawn over those data points which were rejected by the fitting procedure. A lightgray band for $E_{\text {avail }}<1.04 \mathrm{MeV} /$ nucleon delimits the region III from fusion regions I + II. Inset shows by darkgray circles those data points which survived the rejection through the iterative fitting procedure.

We present here the results obtained with the Shen formula [16] while an almost identical result (within a few per mill) obtained when $\sigma_{F}$ is normalized by the $\sigma_{R}$ due to Tripathi [17] will be presented elsewhere [13]. ${ }^{2}$

Figure 1 displays normalized $\mathrm{CF}+\mathrm{IF}$ fusion-evaporation cross sections $\sigma_{N}=\sigma_{F} / \sigma_{R}$ for the 48 systems studied as a function of $E_{\text {inc }}$ in the laboratory reference frame in the spirit of ref. [12]. Data are sorted by the increasing $A_{\text {sys }}$ and systems are differentiated by diverse symbols and a color code (online). The same symbol and color code is used for a given system throughout this letter. As expected, $\sigma_{N}$ decreases with increasing $E_{\text {inc }}$. Moreover, with the normalization applied to fusion cross sections, most of the systems gather in a single arc-like zone: $\sigma_{N}$ from a maximum of $\sim 0.85-0.95 \sigma_{R}$ occurring at $E_{\text {inc }} \approx 3 A-4 A$ $\mathrm{MeV}$ gradually decreases and extincts somewhere about $50 A \mathrm{MeV}$ but it extends up to $80 \mathrm{~A} \mathrm{MeV}$ for the highly mass asymmetric ${ }^{12} \mathrm{C}+{ }^{124} \mathrm{Sn}$ system [68].

In order to further gather the data points we express energy in units of the so-called (system) available energy, i.e. center-of-mass energy per nucleon

$$
E_{\text {avail }}=\frac{E_{\text {c.m. }}}{A_{\text {sys }}}=\frac{E_{\text {lab }}}{A_{\text {prj }}} \frac{A_{\text {prj }} A_{\text {trg }}}{\left(A_{\text {prj }}+A_{\mathrm{trg}}\right)^{2}} .
$$

\footnotetext{
${ }^{2}$ In this long publication we are going to also present a complete table of the raw experimental fusion cross section values as well as those of the calculated reaction cross sections.
} 
Table 1: Values of the best fit coefficients $a, b$, and $c$ and their uncertainties $\Delta a, \Delta b$, and $\Delta c$ of a homographic function (eq. (4)) obtained with the MINUIT package of the CERN library [70]. The first row is for the 238 complete $(\mathrm{CF})$ plus incomplete (IF) fusion evaporation-residue data and the second for the subset of $60 \mathrm{CF}$ data from those experiments in which the contribution of $\mathrm{CF}$ and IF fusion components have been explicitly reported, respectively. data and allow to the fit procedure to iteratively exclude from the next step of fitting those data points which are outside the best fit result by more than three times the corresponding experimental error bar ${ }^{4}$. The consequence of the iterative procedure is a slightly improved description of the mid-region of the arc with no change at its extrema. A stable result has been achieved in four iterations and it is displayed in fig. 2 by the thick red curve. Inset shows

\begin{tabular}{lccc}
\hline Data set & $a \pm \Delta a$ & $b \pm \Delta b$ & $c \pm \Delta c$ \\
\hline Sum of CF and IF fusion & $-0.0925 \pm 0.0019$ & $1.278 \pm 0.029$ & $0.629 \pm 0.034$ \\
CF fusion component only & $-0.217 \pm 0.025$ & $1.43 \pm 0.12$ & $0.419 \pm 0.098$ \\
\hline
\end{tabular}

Indeed, by scaling the abscissa in the units of the available energy one does express the mass asymmetric systems on the same footing with those which are mass symmetric. Such a coordinate scaling greatly ease the comparison of systems of the different asymmetry $\alpha$. The obtained result is displayed in fig. 2. On that manner, as a function of $E_{\text {avail }}$, irrespectively of the individual characteristics of a given reaction system, an overwhelming majority of data follow the same trend. Note, however, the data are very unevenly distributed along the arc. The density of points is much greater for $E_{\text {avail }} \lesssim 3 \mathrm{MeV} /$ nucleon with a kind of stack of the data for $E_{\text {avail }}<1.5 \mathrm{MeV} /$ nucleon. Quite on the contrary, for $E_{\text {avail }}>5 \mathrm{MeV} /$ nucleon fusion data are very scarce. A similar conclusion on the experimental coverage of fusion as a function of energy may be drawn from fig. 1.

Let us interpret the observation that $\sigma_{N}$ data as a function of energy gather along an arc. In a first approximation $\sigma_{F}$ and $\sigma_{R}$ may be expressed by a common functional form

$$
\sigma(E)=\pi r^{2}\left(1-\frac{V}{E}\right)
$$

in which the cross section depends inversely on energy $E$ while the effective radius $r$ and the strength of interaction potential $V$ may be considered constants for a given system (see e.g. refs. $[2,16]){ }^{3}$ In this approximation $\sigma_{N}$ reads

$$
\sigma_{N}\left(E_{\text {c.m. }}\right)=\frac{a_{1}+b_{1} / E_{\text {c.m. }}}{a_{2}+b_{2} / E_{\text {c.m. }}}
$$

where $a_{i}$ and $b_{i}$ are constants. Eq. (3) is equivalent to a homographic function in energy and which we take to be our probe-function

$$
f(x)=a+\frac{b}{c+x},
$$

with $x=E_{\text {avail }}$ and $a, b$, and $c$ the fit parameters.

We apply the above functional relation between normalized cross section and energy to collected experimental fusion data. We start with the full ensemble of $\sigma_{N}$

\footnotetext{
${ }^{3}$ Eq. (2) has been introduced by Bass to describe energy dependence of complete fusion and afterwards has been largely used to describe both complete and incomplete fusion excitation functions.
}

by darkgray circles those data points which survived the iterative fitting procedure. The complement to the full ensemble of $\sigma_{N}$ data, i.e. those data points which were rejected by fitting, are made visible in the main panel of fig. 2 by thin darkgray circles drawn over them.

Speaking of uncertainty in absolute cross sections it is instructive to examine the ${ }^{28} \mathrm{Si}+{ }^{28} \mathrm{Si}$ data (green diamonds). In five experiments 22 fusion cross section values are reported [42-46]. Some of them, taken at the same or very close $E_{\text {inc }}$, differ substantially (see in fig. 1 b)). These discrepancies give an insight into the limits of the precision in deducing the fusion cross section absolute values experimentally and may explain why about $25 \%$ of the data points are removed by the fitting procedure. The group of 16 rejected data points with $E_{\text {avail }} \gtrsim 2.3 \mathrm{MeV} /$ nucleon lying well above the general data trend and which were all measured in seventies of the last century is puzzling. A careful examination of these works could not identify an obvious error or inconsistency in data taking and analysis. Forty years ago, however, the knowledge on deep inelastic process has been far less complete. Without a coincidence measurement it is rather difficult to unambiguously separate fusion-like mechanisms from deep inelastic collisions that involve large transfer of momentum and which may significantly affect the $\sigma_{F}$. The most likely, these 16 data suffer an incorrectly assigned $\sigma_{F}$ value, thus calling to remeasure them [13]. ${ }^{5}$ The fit parameters are reported in table 1. From them one infers the value of the vanishing energy of incomplete fusion process at $E_{\text {avail }}=13.2 \pm 0.6$ $\mathrm{MeV} /$ nucleon $^{6}$. The uncertainty on the fit parameters is

\footnotetext{
${ }^{4}$ Error on fusion cross section $\Delta \sigma_{F}$ is generally in the range of $0.05-0.15 \sigma_{F}$. In case of a non-reported experimental error it has been assumed to be $20 \%$ of the corresponding $\sigma_{F}$ value. No error on $\sigma_{R}$ is considered.

${ }^{5}$ Regarding the ${ }^{14} \mathrm{~N}+{ }^{12} \mathrm{C}$ system, we remind the reader that in their study of the fusion excitation function for 54 individual fusion reactions in the $\sigma_{F}$ vs. $1 / E_{\text {c.m. }}$. representation Giordano et al. [8] have achieved very satisfactory fit to all reactions over the whole available data set except for the portion of the ${ }^{14} \mathrm{~N}+{ }^{12} \mathrm{C}[18,19]$ data with $E_{\text {c.m. }} / A>1.8 \mathrm{MeV} /$ nucleon ( $c f$. in fig. 1 of ref. [8]), i.e. for the same points of this reaction which are rejected by the fitting procedure.

${ }^{6}$ When data are normalized with $\sigma_{R}$ of ref. [17] the result is $E_{\text {avail }}=13.2 \pm 0.7 \mathrm{MeV} /$ nucleon
} 


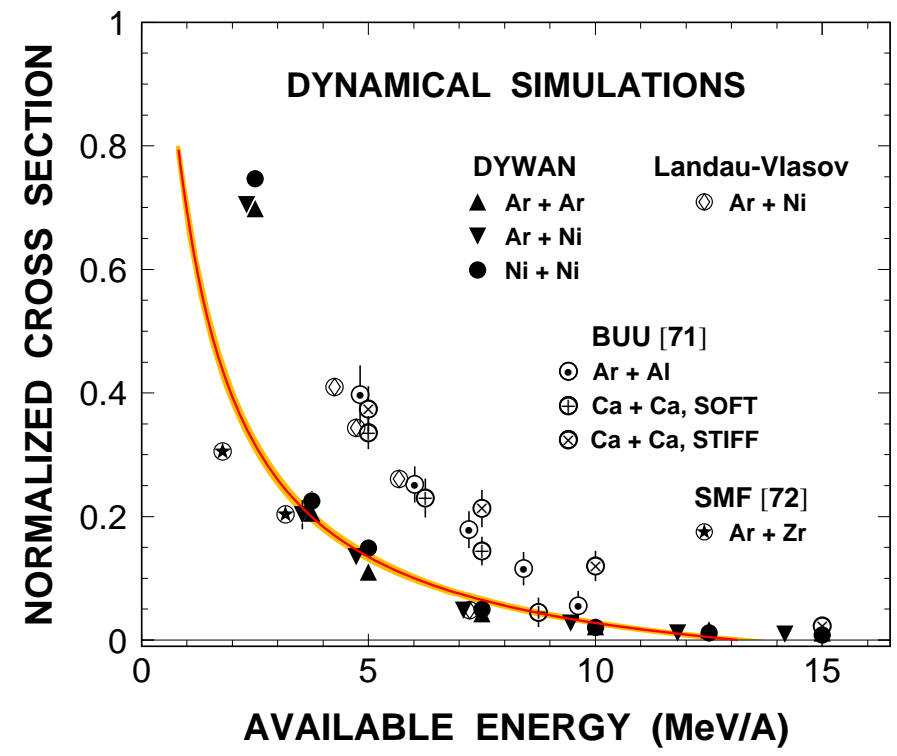

Fig. 3: (Color online.) Prediction of $\sigma_{N}$ as a function of $E_{\text {avail }}$ by dynamical simulations. Full symbols are calculated with the DYWAN model (Zamick soft EOS and the isospin- and energy-dependent phenomenological $\sigma_{\mathrm{NN}}$ ) for the $\mathrm{Ar}+\mathrm{Ar}, \mathrm{Ni}$, and $\mathrm{Ni}+\mathrm{Ni}$ reactions. When plotted, the error bars represent extrema over several independent simulations. Encircled diamonds stand for the Landau-Vlasov simulation of the $\mathrm{Ar}+\mathrm{Ni}$ reaction (Gogny G1D1 EOS and the isospin- and energydependent phenomenological $\left.\sigma_{\mathrm{NN}}\right)$. The BUU simulation results are presented by encircled: point $(\mathrm{Ar}+\mathrm{Al}$, soft $\mathrm{EOS}$, $\left.\sigma_{\mathrm{NN}}=50 \mathrm{mb}\right)$, plus sign $\left(\mathrm{Ca}+\mathrm{Ca}\right.$, soft EOS, $\left.\sigma_{\mathrm{NN}}=41 \mathrm{mb}\right)$, and cross $\left(\mathrm{Ca}+\mathrm{Ca}\right.$, stiff EOS, $\left.\sigma_{\mathrm{NN}}=41 \mathrm{mb}\right)$, respectively [71]. The result of the SMF-Langevin type simulation of the Ar $+\mathrm{Zr}$ system is shown by encircled star symbols [72]. Theoretical fusion cross sections are normalized on the same way as experimental ones. Curve is the fit function from fig. 2 .

rather small (see the narrow orange band around the best fit curve in fig. 2) and consequently that on the energy of disappearance is not too large either.

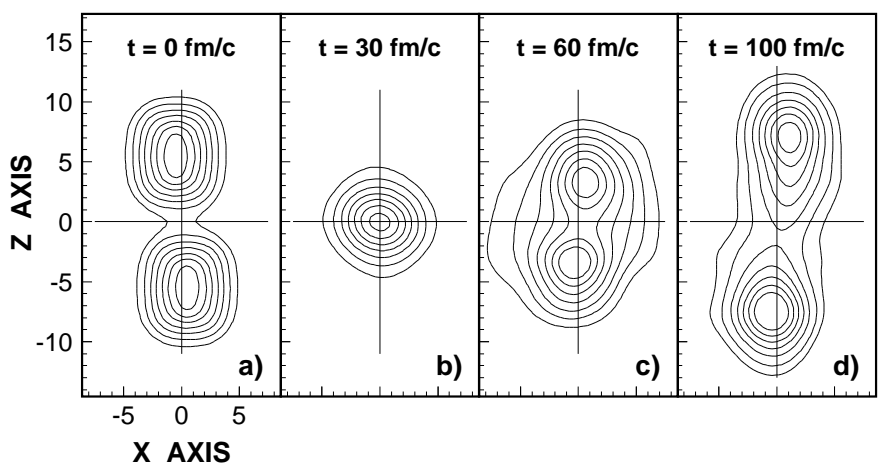

Fig. 4: Density-profile contours in the center-of-mass reference frame projected onto the reaction plane for the $\mathrm{Ni}+\mathrm{Ni} \mathrm{DY}$ WAN simulation at $60 \mathrm{~A} \mathrm{MeV}$ and $b=0.9 \mathrm{fm}$ at a) contact $(t=0 \mathrm{fm} / c), \mathrm{b})$ maximal density $(30 \mathrm{fm} / c)$, c) starting of separation $(60 \mathrm{fm} / c)$, and $\mathrm{d})$ separation $(100 \mathrm{fm} / c)$. The $z$-axis is along the projectile direction.
Theoretical predictions. - Theoretical works on fusion at energies of region III have mostly been devoted to a qualitative study of the phenomenon with little predictions on fusion cross section values. A rare example of the published quantitative studies of fusion excitation function by microscopic transport theories is a simulation of the $\mathrm{Ar}+\mathrm{Al}$ and $\mathrm{Ca}+\mathrm{Ca}$ reactions within the semiclassical BUU model [71]. These results are displayed by encircled point, plus sign, and cross symbols, respectively in fig. 3. Neither the shape nor its absolute value follow the common data trend reproduced by our universal homographic function. Our own unpublished calculation relying on the semiclassical Landau-Vlasov model [73] predicts fusion values comparably far from the universal behavior of the scaled fusion excitation function (see encircled diamonds in fig. 3). Fusion cross sections for the ${ }^{36} \mathrm{Ar}+{ }^{96} \mathrm{Zr}$ system at $9 \mathrm{~A}$ and $16 \mathrm{~A} \mathrm{MeV}$ have recently been investigated using the Langevin force in order to describe density fluctuations [72] (see encircled star symbols in fig. 3). At higher investigated energy the model predicts a value close to the experimental data while at $9 \mathrm{~A} \mathrm{MeV}$ the simulation underestimates our universal law. A study extended to other energies and systems would be necessary before a conclusion on the success of this approach.

It was, thus, a challenge to address this basically onebody problem by means of the DYWAN model, a microscopic approach describing the dynamics of heavy-ion collisions at intermediate energies [74]. The DYWAN model solves an extended TDHF equation by projecting single particle wave functions onto a wavelet basis. The resulting one-body density matrix is a superposition of the Slater determinants of wavelets. Simulation results of fusion cross section for the $\mathrm{Ar}+\mathrm{Ar}, \mathrm{Ar}+\mathrm{Ni}$, and $\mathrm{Ni}+\mathrm{Ni}$ reactions are shown by filled symbols in fig 3 . The scaled fusion excitation function is fairly well predicted for $E_{\text {avail }} \gtrsim 3.75$ $\mathrm{MeV} /$ nucleon (for mass symmetric systems that means for $\left.E_{\text {inc }} \gtrsim 15 A \mathrm{MeV}\right)$. The DYWAN model reproduces both dependence on energy for a given system as well as homographic universality of scaled excitation function. General fusion data is not well reproduced at lower energies. That is not surprising since at this range of energy the model attains its validity limits.

Figure 4 displays the DYWAN density projected onto the reaction plane at selected times of the $\mathrm{Ni}+\mathrm{Ni}$ reaction. From fig. 4 one infers that at $60 \mathrm{~A} \mathrm{MeV}$ in central collisions reaction system promptly reseparates giving rise to a binary exit channel of the dynamically induced fission-like process. The laps of time between the contact and the maximal compression (panels a) and b) in fig. 4, respectively) is roughly a third of the time which system takes to split after reaching its the most compact stage (panels b) and d) in fig. 4). Consequently, the nuclear transparency, i.e. an insufficient stopping power seems to be at the origin of the vanishing of the fusion reaction mechanism. The fusion extinction may be interpreted in terms of a longer mean free path at the Fermi energy region due to the interplay between a weakened nuclear mean field and a 
still insufficient stopping efficiency of nucleon-nucleon collisions. This feature has been several times discussed in the past [75-78] and has recently received an experimental confirmation [79].

Complete and incomplete fusion excitation functions. - Only twelve experiments have explicitly been designed to measure both $\mathrm{CF}$ and IF fusion components. The resultant $60 \mathrm{CF} \sigma_{N}$ belonging to 14 systems are normalized on the same way as previously studied $\mathrm{CF}+\mathrm{IF}$ excitation function and are plotted in fig. 5 as a function of $E_{\text {avail }}$. The same fitting applied to these 60 points resulted in the best fit curve shown by the darkred full curve. In the fitting procedure 3 points were excluded only, all of them from those measurements the IF data of which were excluded be the fitting in fig. 2. These points are marked by lighter colors of the corresponding symbols. In the case of CF the best fit homographic function steeply decreases and the complete fusion reaction mechanism vanishes at $E_{\text {avail }}=6.2_{-1.1}^{+1.3} \mathrm{MeV} /$ nucleon. Unlikely to the best fit of $\mathrm{CF}+\mathrm{IF}$ data the uncertainty on the CF fit coefficients is considerably larger (see table 1) which reflects itself in a larger uncertainty on the energy of CF disappearance.

Inset in fig. 5 shows $\sigma_{N}$ data vs. $1 / E_{\text {avail }}$. This presentation is inspired by the usual way of showing (non-scaled) fusion excitation functions in the $\sigma_{F}$ vs. $1 / E_{\text {c.m. }}$. plane in which, owing to eq. (2), the three regions of fusion are represented by linear segments (see e.g. in refs. $[2,36]$ ). Our scaling brings the different systems to lie, on the average, on a common curve made of two segments of the homographic function and not of two straight lines. In the inset the fusion regions II and III are clearly distinct and the limit between them occurs at $E_{\text {avail }} \approx 1.04 \mathrm{MeV} /$ nucleon independently of the system studied. The fits are carried out on the same manner as before for each fusion region. In the inset, darkgreen dash-dotted and almost horizontal curve fits the region II, whereas the region III is fitted by the darkred full curve, i.e. the same as in the main panel in fig. 5. The existence of these two regions of fusion is emphasized in fig. 2 by the lightgray band for $E_{\text {avail }}<1.04$ $\mathrm{MeV} /$ nucleon.

It is established that the $\mathrm{CF}$ reaction mechanism ceases to exist owing to the dynamical pre-equilibrium emission [11] and CF is progressively replaced by IF. For CF, the center-of-mass energy and the excitation energy $E^{*}$ are up to reaction Q-value equivalent (see short-dashed darkred curve in fig. 5). One infers that an energy of 6 $\mathrm{MeV} /$ nucleon, i.e. a value considerably lower than the average nucleonic binding energy, can at most be deposited into a light compound nucleus produced via heavy-ion collision. Higher values are precluded owing to the dynamical release of excitation energy. It would be very interesting to investigate whether this limit changes for substantially heavier systems.

The established homographic law of the region III of fusion for both CF (full darkred curve in fig. 5) and CF + IF (red full (long-dashed) curve in fig. 2 (fig. 5)) is quite

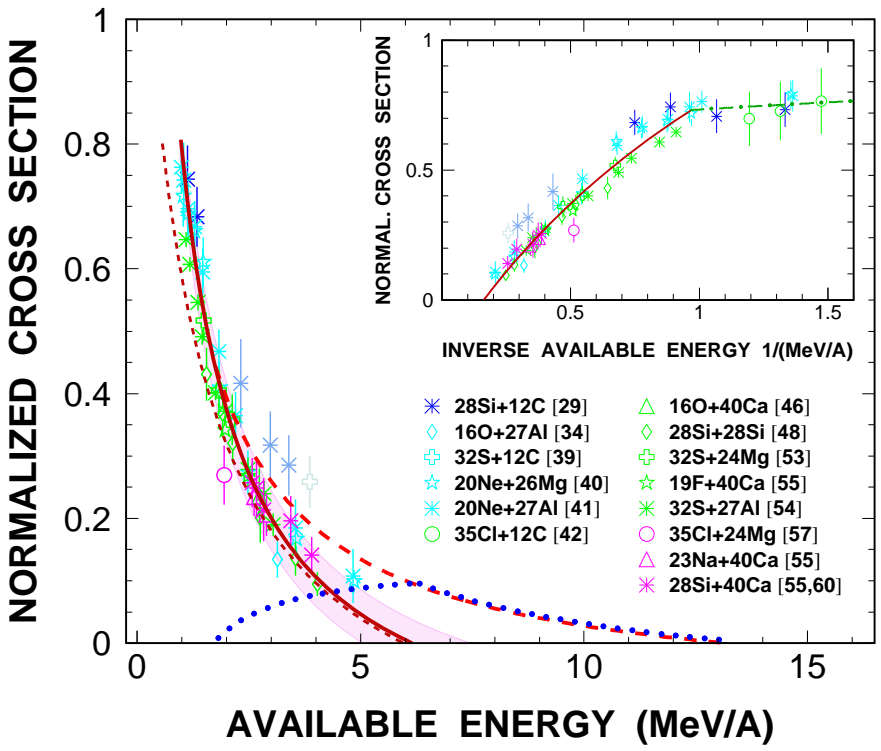

Fig. 5: (Color online.) Complete fusion $\sigma_{N}$ as a function of $E_{\text {avail }}$ (main panel) and as a function of the inverse of the same energy (inset) from those measurements which have reported data on both complete and incomplete fusion. The fit with the homographic function (eq. (4)) in the fusion region III is shown as full darkred curve in both panels. The darkgreen dash-dotted curve in inset is due to the same kind of fit to the fusion region II. The background band around the best fit curve in the main panel is due to the errors on the fit parameters. Red dashed curve is the best fit curve from fig. 2. Blue dotted curve is the difference of both. Short-dashed darkred curve is due to the same kind of fit of the system excitation energy, i.e. $E_{\text {avail }}$ corrected for reaction Q-value per nucleon.

general and it imparts the average dependence of fusion on $E_{\text {avail }}$. Subtracting the two best fit curves allows to infer the main features of IF excitation function (blue dotted curve in fig. 5). IF process opens at $E_{\text {avail }}=1.6$ $\mathrm{MeV} /$ nucleon, at about $E_{\text {avail }}=4.3 \mathrm{MeV} /$ nucleon it contributes equally to the total fusion cross section as $\mathrm{CF}$, each of them amounting for about $0.08 \sigma_{R}$, at the energy of vanishing $\mathrm{CF}$, i.e. at $E_{\text {avail }}=6.2 \mathrm{MeV} /$ nucleon IF attains the maximum of almost $0.1 \sigma_{R}$ and slowly extincts until it vanishes at $E_{\text {avail }}=13.2 \mathrm{MeV} /$ nucleon. For mass symmetric systems, the above vanishing fusion energies correspond to $E_{\mathrm{inc}}$ of about $25 \mathrm{~A}$ and $53 \mathrm{~A} \mathrm{MeV}$, respectively.

Within the accuracy of experimental fusion cross sections and the reliability of theoretical reaction cross sections the scaled fusion excitation function is reaction system independent. Since the extracted properties of the complete and incomplete components of fusion are affected neither by the system mass $A_{\text {sys }}$, mass asymmetry $\alpha$ nor by the $N_{\text {sys }} / Z_{\text {sys }}$ content, they may be considered as universal.

Summary. - To summarize, the systematics of evaporation-residue fusion cross sections far above the Coulomb barrier allowed us to infer a rather universal ho- 
mographic description (eq. (4)) of properly scaled complete and incomplete fusion excitation functions. From our analysis follows that the complete (incomplete) fusion vanishes at system available energy of 6 (13) MeV/nucleon and that incomplete fusion component amounts at most $10 \%$ of total reaction cross section. The total fusion excitation functions of the $\mathrm{Ar}+\mathrm{Ar}, \mathrm{Ar}+\mathrm{Ni}$ and $\mathrm{Ni}+\mathrm{Ni}$ systems at incident energies higher than $\sim 15 \mathrm{~A} \mathrm{MeV}$ are well reproduced by the DYWAN microscopic model. The simulation results suggest that the nuclear transparency is at the origin of fusion disappearance.

Z.B. gratefully acknowledge the hospitality of the Faculté des sciences of Université de Nantes and Laboratoire Subatech.

\section{REFERENCES}

[1] Birkelund J. R. et al., Phys. Rep., 56 (1979) 107.

[2] BAss R., Nuclear Reactions with Heavy Ions (Springer, Berlin) 1980.

[3] Swiatecki W. J., Nucl. Phys. A, 376 (1982) 275.

[4] Birkelund J. R. and Huizenga J. R., Ann. Rev. Nucl. Part. Sci., 33 (1983) 265.

[5] Fröbrich P., Phys. Rep., 116 (1984) 337.

[6] Charity R. J. et al., Nucl. Phys. A, 457 (1986) 441.

[7] Fröbrich P. and Gontchar I. I., Phys. Rep., 292 (1998) 131.

[8] Giordano R. et al., Il Nuovo Cimento A, 103 (1990) 47.

[9] Dasgupta M. et al., Ann. Rev. Nucl. Part. Sci., 48 (1998) 401 and references therein.

[10] URL: http://flerovlab.jinr.ru/flnr/knowledge_base.html and references therein.

[11] Morgenstern H. et al., Phys. Rev. Lett., 52 (1984) 1104.

[12] Lautesse P. et al., Eur. Phys. J. A, 27 (2006) 349.

[13] Eudes P. et al., in preparation.

[14] Eudes P. et al., in Proceedings of the 11th International Conference on Nucleus-Nucleus Collisions, edited by Li BaO-An and Natowitz J., J. Phys.: Conf. Series 420 (IOP Publishing, London) 2013, paper 012133; Eudes P. et al., in Proceedings of the IWM2011 - International Workshop on Multifragmentation and related topics, edited by Frankland J. D. et al. (Italian Physical Society, Bologna) Conf. Proc., 105 (2012) 181.

[15] Kox S. et al., Phys. Rev. C, 35 (1987) 1678.

[16] Shen W. et al., Nucl. Phys. A, 491 (1989) 130.

[17] Tripathi R. K. et al., NASA Technical Paper Nb. 3621 (1997).

[18] Gomez del Campo J. et al., Phys. Rev. C, 19 (1979) 2170.

[19] Stokstad R. G. et al., Phys. Lett. B, 70 (1977) 289.

[20] Shapira D. et al., Phys. Rev. C, 28 (1983) 1148.

[21] Samri M. et al., Phys. Rev. C, 65 (2002) 061603(R).

[22] Natowitz J. B. et al., Phys. Rev. C, 6 (1972) 2133.

[23] Tabor S. L. et al., Phys. Rev. C, 17 (1978) 2136.

[24] Vineyard M. F. et al., Phys. Rev. C, 47 (1993) 2374.

[25] Harmon B. A. et al., Phys. Rev. C, 34 (1986) 552.

[26] Namboodiri M. N. et al., Phys. Rev. C, 11 (1975) 401.

[27] Back B. B. et al., Nucl. Phys. A, 285 (1977) 317.

[28] Kozub R. L. et al., Phys. Rev. C, 11 (1975) 1497.

[29] Gilfoyle G. P. et al., Phys. Rev. C, 46 (1992) 265.
[30] Kolata J. J. et al., Phys. Rev. C, 32 (1985) 1080.

[31] Arena N. et al., Il Nuovo Cimento A, 100 (1988) 953.

[32] Arena N. et al., Phys. Rev. C, 44 (1991) 1947.

[33] Giordano R. et al., Il Nuovo Cimento A, 77 (1983) 135.

[34] Pirrone S. et al., Phys. Rev. C, 64 (2001) 024610.

[35] Lehr H. et al., Nucl. Phys. A, 415 (1984) 149.

[36] Morgenstern H. et al., Z. Phys. A, 313 (1983) 39.

[37] Pirrone S. et al., Phys. Rev. C, 55 (1997) 2482.

[38] Bеск C. et al., Z. Phys. A, 343 (1992) 309.

[39] Beck C. et al., Phys. Rev. C, 54 (1996) 227.

[40] Vigdor S. E. et al., Phys. Rev. C, 20 (1979) 2147.

[41] Beck C. et al., Phys. Rev. C, 39 (1989) 2202.

[42] DiCenzo S. B. et al., Phys. Rev. C, 23 (1981) 2561.

[43] Vineyard M. F. et al., Phys. Rev. C, 41 (1990) 1005.

[44] Meijer R. J. et al., Phys. Rev. C, 44 (1991) 2625.

[45] Oberstedt A. et al., Nucl. Phys. A, 548 (1992) 525.

[46] Box P. F. et al., Phys. Rev. C, 50 (1994) 934.

[47] Kovar D. G. et al., Bull. Am. Phys. Soc., 22 (1977) 66.

[48] Hinnefeld J. D. et al., Phys. Rev. C, 36 (1987) 989.

[49] Rosner G. et al., Phys. Lett. B, 150 (1985) 87.

[50] Pochodzalla J. et al., Phys. Lett. B, 181 (1986) 33.

[51] Beck C. et al., Eur. Phys. J. A, 2 (1998) 281.

[52] Cavallaro S. et al., Phys. Rev. C, 57 (1998) 731.

[53] Gonthier P. L. et al., Nucl. Phys. A, 411 (1983) 289.

[54] Péter J. et al., Nucl. Phys. A, 593 (1995) 95.

[55] Vineyard M. F. et al., Phys. Rev. C, 45 (1992) 1784.

[56] Bisquer E., Ph.D. Thesis (Université Claude Bernard, Lyon I) 1996.

[57] Carter J. et al., Z. Phys. A, 313 (1983) 57.

[58] Rösch W. et al., Phys. Lett. B, 197 (1987) 19.

[59] Rösch W. et al., Nucl. Phys. A, 496 (1989) 141.

[60] Gentner R. et al., Z. Phys. A, 347 (1993) 117.

[61] Doubre H. et al., Phys. Lett. B, 73 (1978) 135.

[62] Lebreton L. et al., Eur. Phys. J. A, 3 (1998) 325.

[63] Borderie B. et al., Z. Phys. A, 298 (1980) 235.

[64] Stege H. et al., Nucl. Phys. A, 489 (1988) 146.

[65] Agarwal S. et al., Z. Phys. A, 296 (1980) 287.

[66] John B. et al., Phys. Rev. C, 56 (1997) 2582.

[67] Kelly M. P. et al., Phys. Rev. C, 56 (1997) 3201.

[68] Nifenecker H. et al., Nucl. Phys. A, 447 (1985) 533c.

[69] Blachot J. et al., Z. Phys. A, 303 (1981) 85.

[70] James F. and Roos M., Comput. Phys. Commun., 10 (1975) 343.

[71] Xu H. M. et al., Phys. Rev. Lett., 65 (1990) 843; XU H. M., Nucl. Phys. A, 568 (1994) 365.

[72] Shvedov L. et al., Phys. Rev. C, 81 (2010) 054605.

[73] Grégoire C. et al., Nucl. Phys. A, 465 (1987) 317; SÉBille F. et al., Nucl. Phys. A, 501 (1989) 137.

[74] Jounult B. et al., Nucl. Phys. A, 628 (1998) 119; DE LA Mota V. and SÉBille F., Eur. Phys. J. A, 12 (2001) 479; SéBille F. et al., Phys. Rev. C, 76 (2007) 024603.

[75] Remaud B. et al., Phys. Lett. B, 180 (1986) 198.

[76] Suraud E. et al., Prog. Part. Nucl. Phys., 23 (1989) 357.

[77] Eudes P. et al., Phys. Rev. C, 56 (1997) 2003; Basrak Z. and Eudes P., in Proceedings of the Yth International Conference on Clustering Aspects of Nuclear Structure and Dynamics, Rab, Croatia, 1999, edited by KoroliJA M. et al. (World Scientific, Singapore) 2000, p. 316.

[78] Novosel I. et al., Phys. Lett. B, 625 (2005) 26.

[79] Lehaut G. et al., Phys. Rev. Lett., 104 (2010) 232701. 